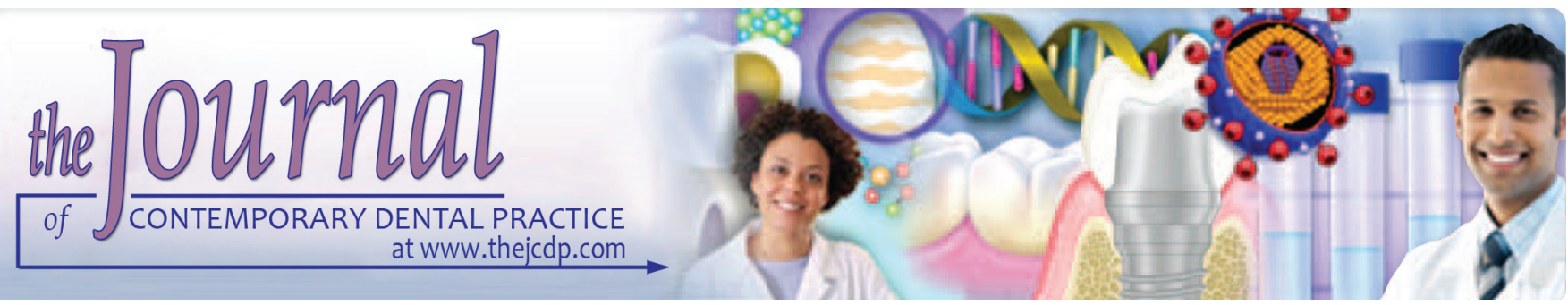

\title{
Nonsurgical Management and 2-year Follow-up by means of Cone Beam Computed Tomography of an Invasive Cervical Resorption in a Molar
}

${ }^{1}$ Mohammed Mashyakhy, ${ }^{2}$ Hemant R Chourasia, ${ }^{3}$ Esam Halboub, ${ }^{4}$ Rafael A Roges, ${ }^{5}$ Gianluca Gambarini

\begin{abstract}
Background: Invasive cervical resorption (ICR) is a relatively uncommon form of external tooth resorption, characterized by an invasive nature. It is usually painless and detection of lesions is often made incidentally. Three-dimensional imaging techniques, such as cone beam computed tomography (CBCT), are useful in the diagnosis and management of ICR as the true extent of the defect cannot always be estimated using conventional radiographs.
\end{abstract}

Aim: The aim of this article is to report on the successful treatment of ICR in mandibular first molar by nonsurgical approach and follow-up by means of CBCT.

Case report: An 18-year-old patient was referred with a complaint of unusual radiolucency in the mesial cervical area of tooth \#19 with unknown etiology. Cone beam computed tomography was performed to assess the extent of the lesion in three spatial levels and diagnosis of Heithersay class III ICR was made. This case presented with ICR (Heithersay class III) on tooth \#19. Nonsurgical root canal treatment and removal of the lesion from the coronal access was performed; the resorptive defect was filled with dual-cure, self-adhesive, resin-modified glass ionomer cement (RMGIC); 6-month follow-up X-ray film showed no changes at the lesion site and tooth was asymptomatic; 1-year follow-up X-ray film showed slight mesial bone loss

\footnotetext{
1,2Department of Restorative Dental Sciences, College of Dentistry, Jazan University, Jazan, Kingdom of Saudi Arabia

${ }^{3}$ Department of Maxillofacial Surgery and Diagnostic Sciences College of Dentistry, Jazan University, Jazan, Kingdom of Saudi Arabia

${ }^{4}$ Department of Endodontics, Herman Ostrow School of Dentistry of USC, Los Angeles, California, USA

${ }^{5}$ Department of Restorative Dentistry and Endodontics, Sapienza University of Rome, Rome, Italy

Corresponding Author: Mohammed Mashyakhy, Department of Restorative Dental Sciences, College of Dentistry, Jazan University, Jazan, Kingdom of Saudi Arabia, Phone: +966557224154, e-mail: dr.mashyakhy@hotmail.com
}

and a probing depth of $3 \mathrm{~mm}$; finally, 2-year follow-up СВCT images showed no recurrence and no further bone destruction at the lesion site.

Conclusion: The intraoral radiographs revealed the resorptive changes in two dimensions; therefore, the actual extent and location of the lesions are not fully understood. On the contrary, CBCT is a very useful tool to achieve a proper diagnosis; it detects the extent of the defect more accurately and hence, improves the treatment outcomes of ICR.

Clinical significance: The ICR is usually seen as a late complication to traumatic injuries of the teeth; it is essential, therefore, that the patients who were exposed to situations that can damage the integrity of periodontal tissue need to have careful periodic recalls and X-ray examinations.

Keywords: Cone beam computed tomography, Invasive cervical resorption, Mandibular first molar, Nonsurgical root canal treatment.

How to cite this article: Mashyakhy M, Chourasia HR, Halboub E, Roges RA, Gambarini G. Nonsurgical Management and 2-year Follow-up by means of Cone Beam Computed Tomography of an Invasive Cervical Resorption in a Molar: A Case Report. J Contemp Dent Pract 2018;19(9):1152-1156.

Source of support: Nil

Conflict of interest: None

\section{BACKGROUND}

Invasive cervical resorption is a relatively uncommon form of external tooth resorption, characterized by an invasive nature. It starts below the epithelial attachment and subsequently invades the cementum, dentin, and, in late stage, reaches the pulp. The exact etiology of ICR is unknown; however, several potential predisposing factors have been suggested, which include history of orthodontic treatment, trauma, and intracoronal bleaching. ${ }^{1,2}$ The condition is painless and detection of lesion is often made incidentally during routine examination 
of radiographs. The resorption rarely penetrates the pulp because of the presence of so-called pericanalar resorption-resistant sheet. ${ }^{3}$ Two-dimensional conventional radiograph like intraoral periapical (IOPA) may show a cloudy appearance in the cervical area with poorly defined border in the early stage of the lesion. In late stage, however, the radiolucency extends coronally and apically with an irregular shape, but the canal can be traced, which indicates that the lesion is external. ${ }^{2,4}$ Three-dimensional imaging techniques, such as CBCT, are useful in the diagnosis and management of ICR, as the true extent of the defect cannot always be estimated using conventional radiographs. ${ }^{5}$

Invasive cervical resorption and external cervical resorption are commonly used terms to describe the same condition; Heithersay ${ }^{6}$ preferred the term ICR due to its invasive and aggressive nature. Heithersay ${ }^{7}$ developed a clinical classification of ICR according to the degree of the damage to the mineralized tissues of the tooth into four categories. Class I corresponds to a small invasive resorptive lesion near the cervical area with shallow penetration into dentin, class II represents a well-defined resorptive lesion close to the coronal pulp chamber with little or no extension into the radicular dentin, class III represents a deeper invasion of dentin not only involving the coronal dentin but also extending at least to the coronal third of the root, and class IV corresponds to a resorptive defect extending beyond the coronal third of the root canal and may involve almost the entire root.

Different approaches have been suggested for the treatment of ICR. The treatment aims to completely remove and inactivate the resorptive lesion, and to restore the defect with suitable restorative material. This case report presents CBCT-based early diagnosis, assessment, nonsurgical (conservative) treatment approach, and 2-year follow-up documentation of Heithersay class III ICR in a mandibular first molar.

\section{CASE REPORT}

An 18-year-old Hispanic female patient was referred by her orthodontist to the Advanced Endodontic Program, Herman Ostrow School of Dentistry, University of Southern California with a complaint of unusual radiolucent area in the mesial cervical area of tooth \#19. The orthodontist noticed the lesion on routine IOPA radiograph before placing orthodontic band. The patient's medical history was noncontributory and no history of trauma was reported. Intraoral examination revealed class I amalgam and composite restorations on the occlusal surface of \#19. After consultation with the orthodontist, it was explained that the composite restoration was placed to raise the bite. Cold test revealed normal pulp response and percussion test reported normal periapical area. Probing depth of $2-3 \mathrm{~mm}$ around the tooth was noted with healthy gingiva. The IOPA radiograph (Fig. 1A) showed radiolucent lesion in the cervical area of the tooth. Cone beam computed tomography (CS 9000 3D Extraoral Imaging System; Carestream Health, Inc.) was performed at $76 \mu \mathrm{m}$ resolution to assess the extent of the lesion in three spatial levels and small field of view $4 \times 4$ was chosen. ${ }^{8}$ Based on the CBCT images and threedimensional reconstructions (Figs $1 \mathrm{~B}$ to $\mathrm{D}$ ), a diagnosis of Heithersay class III ICR was made. The patient and orthodontist were informed about the diagnosis, treatment options, and prognosis of the tooth, and informed consent was obtained. Nonsurgical root canal treatment and removal of the lesion from the coronal access were planned as less invasive procedure rather than surgical approach.

Two carpules of Lidocaine local anesthesia (72 mg) $0.036 \mathrm{mg}$ of epinephrine were administered and rubber dam isolation was done. Under dental operative microscope (Zeiss OPMI pico, Carl Zeiss Surgical, Germany) at $8 \times$ magnification, composite and amalgam restorations were removed and the lesion was accessed from coronal approach by using round carbide bur (Fig. 2A). The root canal treatment was subsequently planned because the resorption lacuna was in close relation with mesial root canals. The access cavity was prepared using Endo Access kit (Dentsply Tulsa Dental Specialties; Tulsa, Oklahoma), and the granulation tissue and soft dentin (Figs 2B and C) were removed with spoon excavator (Hu-Friedy, Chicago, USA). Further excavation was done toward the area of entrance of lesion (Fig. 2D) to ensure complete removal of resorptive lesion. To dissolve possible tissue remnants, cotton pellets soaked with $6 \%$ sodium hypochlorite were applied for 3 times each for 1 minute.

Three canals, namely mesiobuccal (MB), mesiolingual (ML), and distal (D), were cleaned and shaped with Vortex rotary files (Dentsply Tulsa Dental Specialties, Johnson City); the master apical file (MAF) was 35/0.04 for $\mathrm{MB}$ and $\mathrm{ML}$, and 40/0.06 for D canal. Intracanal irrigation was performed with $6 \%$ sodium hypochlorite after each instrument. Canal orifices were blocked with gutta-percha (Fig. 2E), and the resorptive defect was filled (Fig. 2F) with dual-cure, self-adhesive, RMGIC (Geristore; Den-Mat Corporation, Santa Maria, CA). Gutta-percha cones were removed, calcium hydroxide (TempCal; Pulpdent Corporation, Watertown, USA) was applied as intracanal medicament, and access cavity was restored with temporary filling material (Cavit; 3M ESPE AG, Seefeld, Germany). The patient presented 1 week later and the tooth was asymptomatic. Gutta-percha cones with sizes corresponding to MAFs were used to fill the 

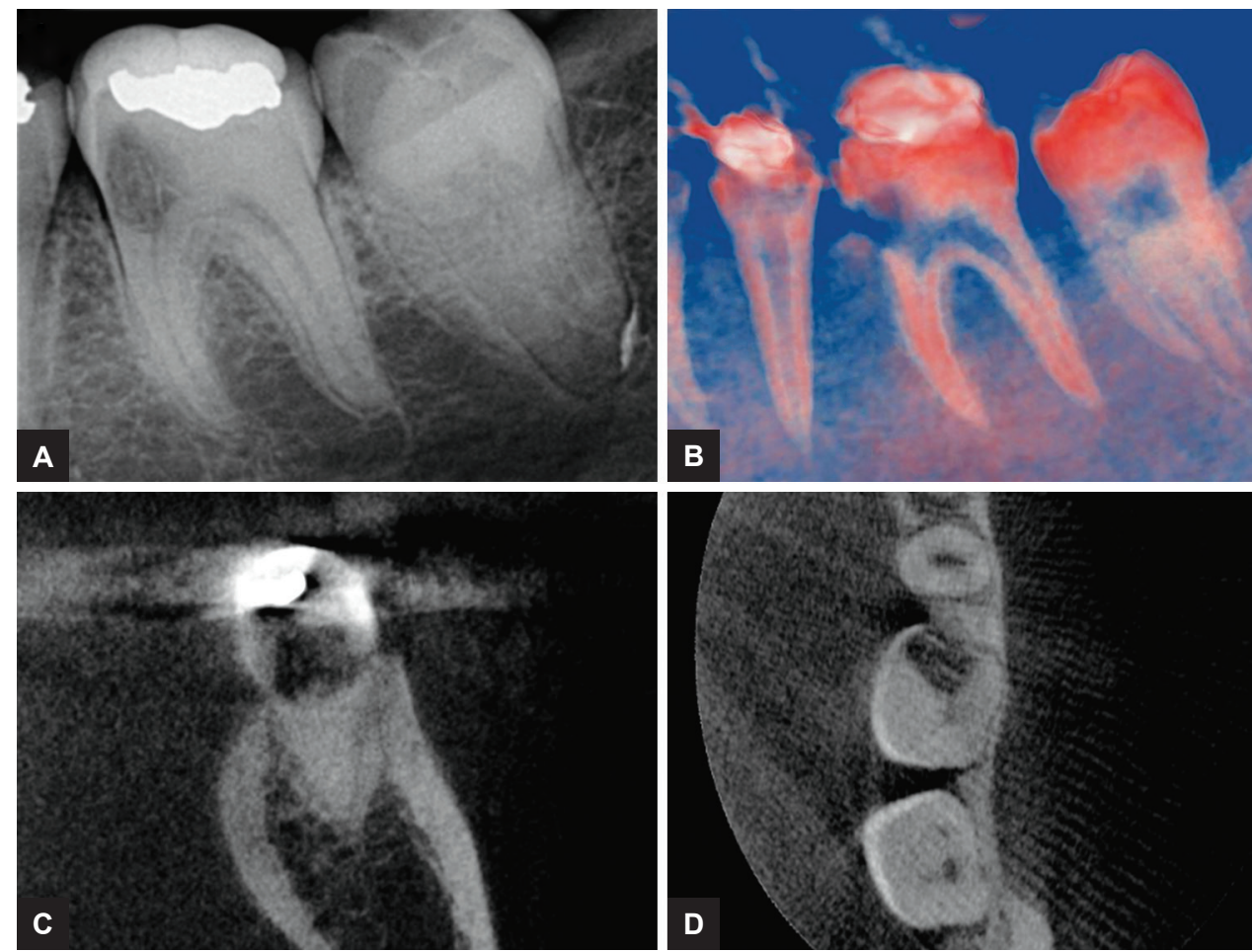

Figs 1A to D: (A) The IOPA radiograph showing resorptive lesion in the cervical region. (B) Sagittal section, color contrast CBCT showing the extension of the lesion into the pulp. (C) Three-dimensional reconstruction showing the extension of the lesion in buccolingual direction. (D) Three-dimensional reconstruction of axial section showing the lesion extension in mesiodistal and buccolingual direction
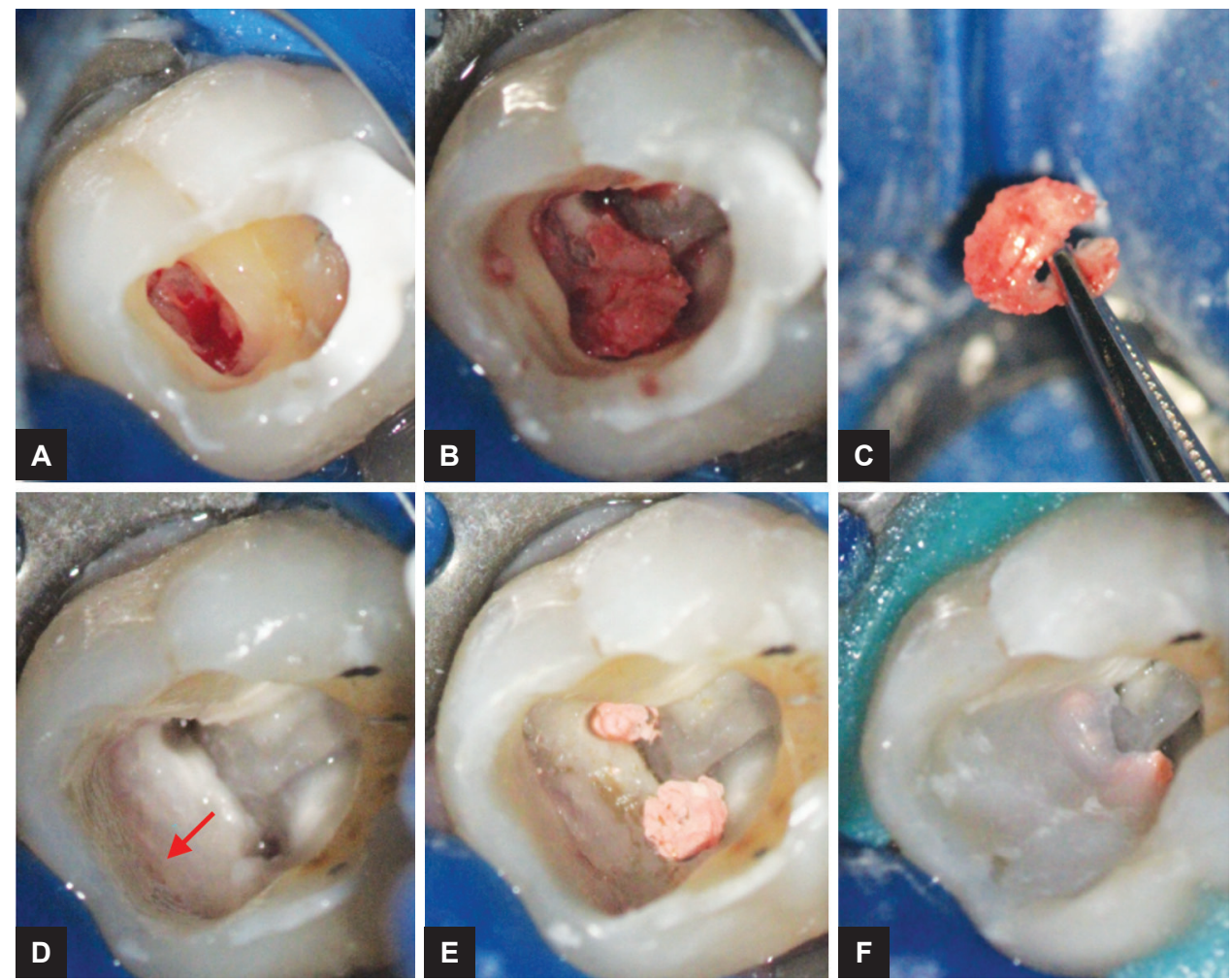

Figs 2A to F: (A) Coronal access to the lesion. (B) The granulation tissue and soft dentin in the cervical region. (C) The granulation tissue and soft dentin removed from the lesion site. (D) Cleaned lesion site showing the site of entrance of the lesion (arrow) and prepared mesial canal orifices. (E) Gutta-percha cones used to block the canal orifices before restoring the defect. $(F)$ Lesion site restored with RMGIC 

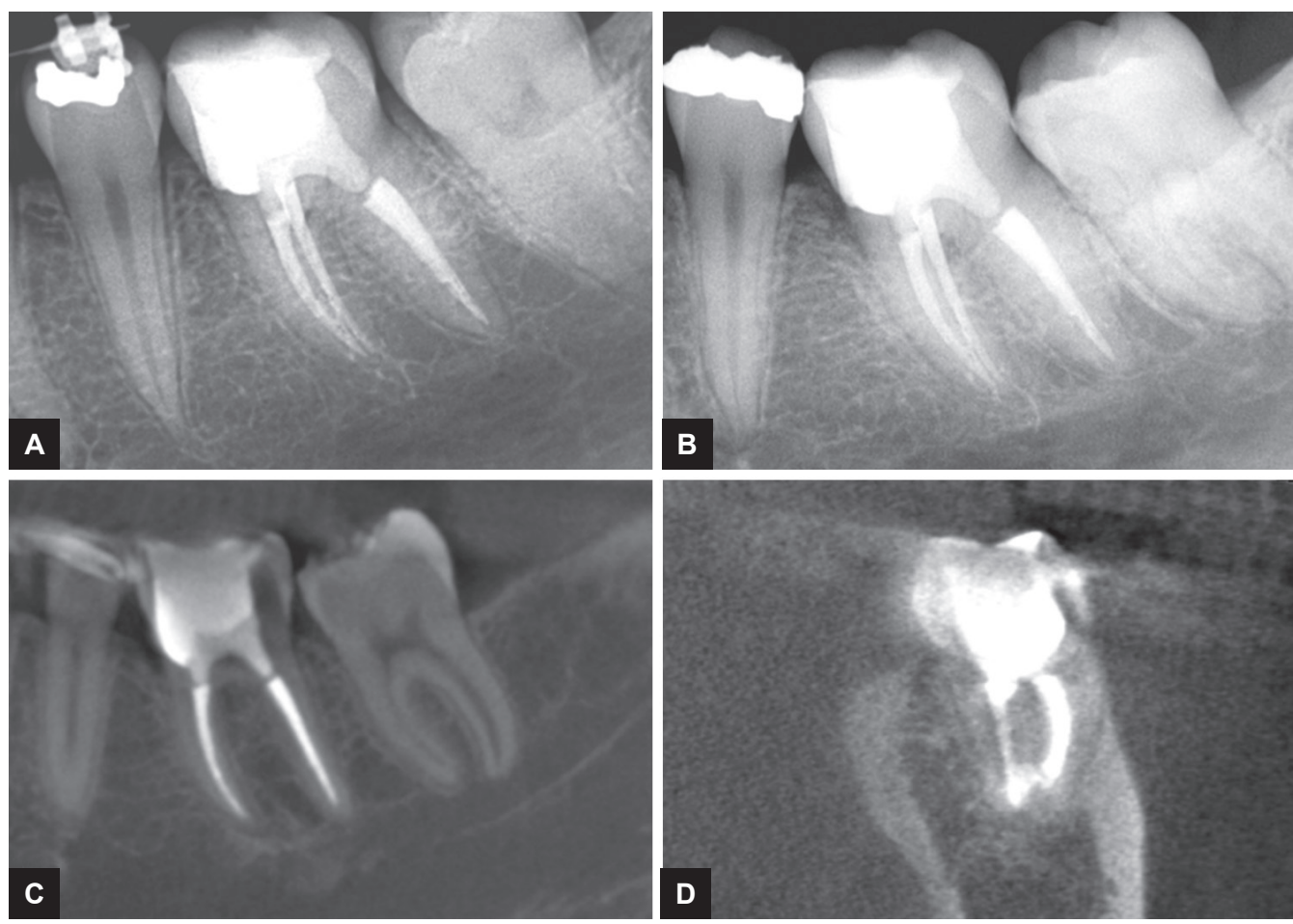

Figs 3A to D: (A) Postoperative IOPA radiograph showing the restored lesion and obturated canals. (B) One-year follow-up IOPA radiograph showing slight mesial bone loss. (C) Two years follow-up CBCT sagittal section showing slight mesial bone loss. (D) Two years follow-up three-dimensional reconstruction showing intact well-adapted restoration

root canals with warm vertical compaction technique and AH Plus sealer (Dentsply Maillefer, Tulsa, Oklahoma). The canals orifices were sealed with glass ionomer (Fugi IX; GC America) while the access cavity was restored with composite restoration and postoperative $x$-film was taken (Fig. 3A). The patient and her orthodontist were advised to wait for 6 months before resuming the orthodontic treatment, as an observation period to analyze the outcome of the treatment. On 6 months recall, tooth \#19 was completely asymptomatic and X-ray film showed no changes at the lesion site. However, the orthodontist continued the treatment before elapsing 6 months, and placed a band on \#19. The patient reported at 1-year follow-up with asymptomatic tooth and completed orthodontic treatment; an IOPAX-ray film (Fig. 3B) showed a slight mesial bone loss with a probing depth of $3 \mathrm{~mm}$. On a 2-year recalled visit, the tooth was asymptomatic and a control CBCT was performed in three spatial levels to further assess the treated site. Both in sagittal and axial section, no reoccurrence of the lesion and no further bone loss were noted (Figs $3 \mathrm{C}$ and D).

\section{DISCUSSION}

In most instances, ICR is seen as a late complication to traumatic injuries of the teeth, orthodontic tooth movement, periodontal treatment, bleaching of teeth, and a wide variety of "traumatic" conditions. Heithersay attributed $24.1 \%$ of ICR to orthodontic treatment as a potential predisposing condition and found that the most commonly affected teeth were maxillary canines, maxillary incisors, and mandibular first molars. ${ }^{1,2}$ It is essential, therefore, that the patients who were exposed to situations that can damage the integrity of periodontal tissue, such as orthodontic treatment, need to have careful periodic recalls and X-ray examinations. The sooner the diagnosis, less complicated the treatment and consequently better the prognosis. The two-dimensional intraoral radiographs revealed the resorptive changes, but the actual extent and location of the lesions are not fully understood. ${ }^{9}$ On the contrary, CBCT is a very useful tool to achieve a proper diagnosis; it detects the extent of the defect more accurately and hence, improves the treatment outcomes of ICR. ${ }^{10}$ In this case, CBCT was used to diagnose, classify, and assess the size and extent of the resorptive defect and to evaluate the treatment outcomes during follow-up visits.

In the present case, the resorption of the mandibular first molar was detected relatively early and graded as class III lesion involving the coronal dentin with extension to the coronal third of the root. Typically, surgical treatment of varying degrees of ICR involves periodontal flap reflection, curettage, granulation tissue removal, and restoration of the defect with suitable material, such as $\mathrm{RMGIC}^{11,12}$ mineral trioxide aggregate (MTA), ${ }^{13,14}$ 
calcium-enriched mixture cement, ${ }^{15}$ and Biodentine, ${ }^{16}$ and repositioning the flap to its original position. In the present case, the resorption lacuna was exposed from the coronal approach rather than surgical exposure by full-thickness mucoperiosteal flaps.

Topical application of an aqueous solution of $90 \%$ trichloroacetic acid was recommended by different authors for the inactivation of the resorbing process. ${ }^{6,17}$ However, in the present case, $6 \%$ sodium hypochlorite was applied and refreshed frequently to dissolve the possible remnants of granulation tissue. Sodium hypochlorite at higher concentrations showed increased efficacy for tissue dissolution, and refreshing the solution at the site resulted in a marked increase of hypochlorite effect. ${ }^{18}$ The root canal treatment was performed as the resorption lacuna was in close relation with the mesial root canals. The RMGIC was used to restore the resorptive defect in the present case. It offers the advantages of glass ionomer and resin; releases fluoride; has an increased resistance to microleakage; adheres to the tooth structure; and is less soluble than the conventional glass ionomer. ${ }^{19,20}$ The MTA has been recommended in several case reports as the restorative material of choice because it has favorable chemical and physical properties, sealing ability, antibacterial activity, and biocompatibility, and it creates an ideal environment for hard tissue healing. ${ }^{21,22}$ However, MTA's beneficial properties are questionable when it is exposed to the oral cavity. ${ }^{23}$ The treatment outcomes were accessed with the follow-up $X$-rays at 6 months, at 1 year, and at 2 years combined with CBCT examination, which revealed satisfactory outcome.

\section{REFERENCES}

1. Heithersay GS. Clinical, radiologic and histopathologic features of invasive cervical resorption. Quintessence Int 1999 Jan;30(1):27-37.

2. Heithersay GS. Invasive cervical resorption: an analysis of potential predisposing factors. Quintessence Int 1999 Feb;30(2):83-95.

3. Mavridou AM, Pyka G, Kerckhofs G, Wevers M, Bergmans L, Gunst V, Huybrechts B, Schepers E, Hauben E, Lambrechts P. A novel multimodular methodology to investigate external cervical tooth resorption. Int Endod J 2016 Mar;49(3):287-300.

4. Tronstad L. Root resorption: etiology, terminology and clinical manifestations. Endod Dent Traumatol 1988 Dec;4(6):241-252.

5. Patel S, Dawood A. The use of cone beam computed tomography in the management of external cervical resorption lesions. Int Endod J 2007 Sep;40(9):730-737.

6. Heithersay GS. Invasive cervical resorption. Endod Topics 2004;7(1):73-92.

7. Heithersay GS. Invasive cervical resorption following trauma. Aust Endod J 1999 Aug;25(2):79-85.
8. AAE and AAOMR Joint Position Statement: use of cone beam computed tomography in endodontics 2015 update. J Endod 2015 Sep;41(9):1393-1396.

9. Kamburoglu K, Barenboim SF, Arıtürk T, Kaffe I. Quantitative measurements obtained by micro-computed tomography and confocal laser scanning microscopy. Dentomaxillofac Radiol 2008 Oct;37(7):385-391.

10. Estevez R, Aranguren J, Escorial A, de Gregorio C, De La Torre F, Vera J, Cisneros R. Invasive cervical resorption Class III in a maxillary central incisor: diagnosis and followup by means of cone-beam computed tomography. J Endod 2010 Dec;36(12):2012-2014.

11. Subramanyappa SK, Parthasarathy B, Manjegowda PG, Rajeev S. Management of perforating invasive cervical resorption: two case reports. J Indian Acad Oral Med Radiol 2012 Dec;24(4):346-349.

12. Tavares WL, Lopes RC, Oliveira RR, Souza RG, Henriques LC, Ribeiro-Sobrinho AP. Surgical management of invasive cervical resorption using resin-modified glass ionomer cement. Gen Dent 2013 Nov-Dec;61(7):e16-e18.

13. Yilmaz HG, Kalender A, Cengiz E. Use of mineral trioxide aggregate in the treatment of invasive cervical resorption: a case report. J Endod 2010 Jan;36(1):160-163.

14. Ikhar A, Thakur N, Patel A, Bhede R, Patil P, Gupta S. Management of external invasive cervical resorption tooth with mineral trioxide aggregate: a case report. Case Rep Med 2013;2013:139801.

15. Utneja S, Nawal RR, Talwar S, Verma M. Current perspectives of bio-ceramic technology in endodontics: calcium enriched mixture cement-review of its composition, properties and applications. Restor Dent Endod 2015 Feb;40(1):1-13.

16. Baranwal AK. Management of external invasive cervical resorption of tooth with biodentine: a case report. J Conserv Dent 2016 May-Jun;19(3):296-299.

17. Fuss Z, Tsesis I, Lin S. Root resorption-diagnosis, classification and treatment chooses based on stimulation factors. Dent Traumatol 2003 Aug;19(4):175-182.

18. Stojicic S, Zivkovic S, Qian W, Zhang H, Haapasalo M. Tissue dissolution by sodium hypochlorite: effect of concentration, temperature, agitation, and surfactant. J Endod 2010 Sep;36(9):1558-1562.

19. Simon J, de Rijk WG. Dental cements. Inside Dentistry 2006;2(2):42-47.

20. Estafan D, Pines MS, Erakin C, Fuerst PF. Microleakage of Class V restorations using two different compomer systems: an in vitro study. J Clin Dent 1999;10(4):124-126.

21. Lee SJ, Monset M, Torabinejad M. Sealing ability of mineral trioxide aggregate for repair of lateral root perforations. J Endod 1993 Nov;19(11):541-544.

22. Koh ET, McDonald F, Pitt Ford TR, Torabinejad M. Cellular response to mineral trioxide aggregate. J Endod 1998 Aug;24(8):543-547.

23. Schwartz RS, Robbin JW, Rindler E. Management of invasive cervical resorption: observations from three private practices and a report of three cases. J Endod 2010 Oct;36(10): 1721-1730. 